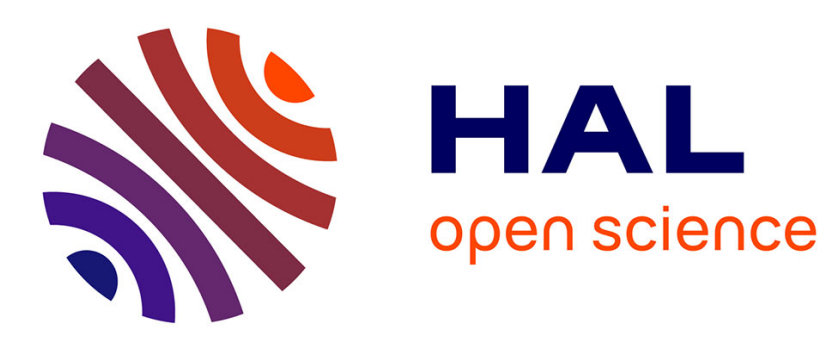

\title{
An ISS based Solution to avoid Local Minima in the Potential Field Method
}

Matteo Guerra, Gang Zheng, Denis Efimov, Wilfrid Perruquetti

\section{To cite this version:}

Matteo Guerra, Gang Zheng, Denis Efimov, Wilfrid Perruquetti. An ISS based Solution to avoid Local Minima in the Potential Field Method. Proc. ECC'15, Jul 2015, Linz, Austria. hal-01140322

\section{HAL Id: hal-01140322 \\ https://hal.inria.fr/hal-01140322}

Submitted on 8 Apr 2015

HAL is a multi-disciplinary open access archive for the deposit and dissemination of scientific research documents, whether they are published or not. The documents may come from teaching and research institutions in France or abroad, or from public or private research centers.
L'archive ouverte pluridisciplinaire HAL, est destinée au dépôt et à la diffusion de documents scientifiques de niveau recherche, publiés ou non, émanant des établissements d'enseignement et de recherche français ou étrangers, des laboratoires publics ou privés. 


\title{
An ISS based Solution to avoid Local Minima in the Potential Field Method
}

\author{
Matteo Guerra, Gang Zheng, Denis Efimov and Wilfrid Perruquetti
}

\begin{abstract}
This work solves the obstacle avoidance problem extending the Potential Field (PF) method for a mobile robot. The usual definition of the PF has been modified to have a field which is continuous everywhere. It is shown that the system has an attracting equilibrium at the target point, repelling equilibriums in the centers of the obstacles and saddle points on the borders. Those unstable equilibriums are avoided capitalizing on the established Input-to-State Stability (ISS) property of this multistable system. To escape a local minima this work makes the most of ISS property that is not lost for perturbations. And for small properly designed disturbances the global attractivity of the target point is proved. The proposed modification of the PF method is shown to be effective by simulation and then applied for unicycle-like mobile robots with additive input disturbances.
\end{abstract}

\section{INTRODUCTION}

Within the most studied topics in the robotic field there is, of course, obstacles avoidance due to the obvious necessity for the platforms to avoid collisions while executing a task. Many solutions have been proposed to tackle the problem. One of the most used is the Potential Field Method, firstly introduced by Khatib in [6]; for motion planning, the Potential Field (PF) drives the robot along a potential field whose minimum is at the target and each obstacle generates an additional repellent force which drives the robot away. It has been shown that this solution, even though mathematically elegant and quite effective practically, has some drawbacks when special events occur [8]. In its standard formulation local minima could appear resulting in a trap for the robot that gets blocked (the easiest example is the case in which the obstacle center belongs to the straight line connecting the target and the robot center); other issues arise if several obstacles are too closely placed or when the size of them are considerably bigger than the robot (i.e. a wall) resulting in oscillations in both cases. A solution was proposed by Koditschek et al. in [13], they proposed navigation functions in a $n$-dimensional space in which the adopted potential field had no other local minima than the target specified, supposing though that the complete environment was known a priori. Other solutions use a harmonic potential field proposed in [2], [14], and the more recent [9], in which the method computes solutions to

Matteo Guerra, Gang Zheng, Denis Efimov and Wilfrid Perruquetti are with Non-A team @ Inria, Parc Scientifique de la Haute Borne, 40 av. Halley, 59650 Villeneuve d'Ascq, France and CRIStAL (UMR-CNRS 9189), Ecole Centrale de Lille, BP 48, Cité Scientifique, 59651 Villeneuve-d'Ascq, France. (matteo.guerra@inria.fr)

D. Efimov is with Department of Control Systems and Informatics, Saint Petersburg State University of Information Technologies Mechanics and Optics (ITMO), 49 av. Kronverkskiy, 197101 Saint Petersburg, Russia.
Laplace's Equation in arbitrary $n$-dimensional domains, and results in a weak form of [13].

Last flaw of the method is the possibility to miss the target in case of an obstacle too close to it. This problem is called Goals nonreachable with obstacles nearby (GNRON). Treated in [4], it deals with the case in which the repulsive force generated by an obstacle close to the target generate a force higher than the attractive one, preventing the robot to accomplish the task.

There are also methods which do not eliminate unwanted equilibriums but generate local forces, Virtual Hill, to escape the disturbing minimum as in [12]. Many other modifications have been proposed in order to overcome all the cited shortages also in the case of the collision avoidance for multiple robots [11], [7], [15].

This work presents a modification of the standard Potential Field method in which first of all, the field is continuous everywhere, property that is not common in literature; considering a particle in 2D dimension, the integrator (that has the gradient of such a field as input) under proper assumptions is shown to be an ISS stable system with respect to decomposable invariants sets using the results presented in [1], while local minima are avoided adding an input which plays on the fact that the appearance of any bounded perturbation does not compromise the ISS property. Simulations are shown to support the theoretical results and the effectiveness for motion planning and collision avoidance considering a particle in 2D dimension. Moreover simulations have been carried for an unicyle-like non-holonomic Wheeled Mobile Robots (WMR). In the case of the WMR a finite-time controller has been designed to regulate the orientation to guarantee a fast and robust response.

\section{Preliminaries}

For an $n$-dimensional $\mathcal{C}^{2}$ connected and orientable Riemannian manifold $M$ without boundary $(0 \in M)$, let the map $f: M \times \mathbb{R}^{m} \rightarrow T_{x} M$ be of class $\mathcal{C}^{1}$ ( $T_{x} M$ is the tangent space), and consider a nonlinear system of the following form:

$$
\dot{x}(t)=f(x(t), d(t))
$$

where the state $x \in M$ and $d(t) \in \mathbb{R}^{m}$ (the input $d(\cdot)$ is a locally essentially bounded and measurable signal) for $t \geq 0$. We denote by $X(t, x ; d(\cdot))$ the uniquely defined solution of (1) at time $t$ fulfilling $X(0, x ; d(\cdot))=x$. Together with (1) we will analyze its unperturbed version:

$$
\dot{x}(t)=f(x(t), 0) .
$$

A set $S \subset M$ is invariant for the unperturbed system (2) if $X(t, x ; 0) \in S$ for all $t \in \mathbb{R}$ and for all $x \in S$. For a set 
$S \subset M$ define the distance to the set $|x|_{S}=\min _{a \in S} \delta(x, a)$ from a point $x \in M$, where the symbol $\delta\left(x_{1}, x_{2}\right)$ denotes the Riemannian distance between $x_{1}$ and $x_{2}$ in $M,|x|=|x|_{\{0\}}$ for $x \in M$ or a usual euclidean norm of a vector $x \in \mathbb{R}^{n}$. For a signal $d: \mathbb{R} \rightarrow \mathbb{R}^{m}$ the essential supremum norm is defined as $\|d\|_{\infty}=\operatorname{ess}_{\sup } \operatorname{sun}_{t \geq 0}|d(t)|$.

\section{A. Decomposable sets}

Let $\Lambda \subset M$ be a compact invariant set for (2).

Definition 1. [10] A decomposition of $\Lambda$ is a finite and disjoint family of compact invariant sets $\Lambda_{1}, \ldots, \Lambda_{k}$ such that

$$
\Lambda=\bigcup_{i=1}^{k} \Lambda_{i}
$$

For an invariant set $\Lambda$, its attracting and repulsing subsets are defined as follows:

$$
\begin{aligned}
& W^{s}(\Lambda)=\left\{x \in M:|X(t, x, 0)|_{\Lambda} \rightarrow 0 \text { as } t \rightarrow+\infty\right\}, \\
& W^{u}(\Lambda)=\left\{x \in M:|X(t, x, 0)|_{\Lambda} \rightarrow 0 \text { as } t \rightarrow-\infty\right\} .
\end{aligned}
$$

Define a relation on $\mathcal{W} \subset M$ and $\mathcal{D} \subset M$ by $\mathcal{W} \prec \mathcal{D}$ if $W^{s}(\mathcal{W}) \cap W^{u}(\mathcal{D}) \neq \oslash$.

Definition 2. [10] Let $\Lambda_{1}, \ldots, \Lambda_{k}$ be a decomposition of $\Lambda$, then

1. An $r$-cycle $(r \geq 2)$ is an ordered $r$-tuple of distinct indices $i_{1}, \ldots, i_{r}$ such that $\Lambda_{i_{1}} \prec \ldots \prec \Lambda_{i_{r}} \prec \Lambda_{i_{1}}$.

2. A 1 -cycle is an index $i$ such that $\left[W^{u}\left(\Lambda_{i}\right) \cap W^{s}\left(\Lambda_{i}\right)\right]-$ $\Lambda_{i} \neq \varnothing$.

3. A filtration ordering is a numbering of the $\Lambda_{i}$ so that $\Lambda_{i} \prec \Lambda_{j} \Rightarrow i \leq j$.

As we can conclude from Definition 2, existence of an $r$ cycle with $r \geq 2$ is equivalent to existence of a heteroclinic cycle for (2) [5]. And existence of a 1-cycle implies existence of a homoclinic cycle for (2) [5].

Definition 3. The set $\mathcal{W}$ is called decomposable if it admits a finite decomposition without cycles, $\mathcal{W}=\bigcup_{i=1}^{k} \mathcal{W}_{i}$, for some non-empty disjoint compact sets $\mathcal{W}_{i}$, which form a filtration ordering of $\mathcal{W}$, as detailed in definitions 1 and 2 .

Let a compact set $\mathcal{W} \subset M$ be containing all $\alpha$ - and $\omega$-limit sets of (2).

\section{B. Robustness notions}

The following robustness notions for systems in (1) have been introduced in [1].

Definition 4. We say that the system (1) has the practical asymptotic gain (pAG) property if there exist $\eta \in \mathcal{K}_{\infty}$ and a non-negative real $q$ such that for all $x \in M$ and all measurable essentially bounded inputs $d(\cdot)$ the solutions are defined for all $t \geq 0$ and the following holds:

$$
\limsup _{t \rightarrow+\infty}|X(t, x ; d)|_{\mathcal{W}} \leq \eta\left(\|d\|_{\infty}\right)+q
$$

If $q=0$, then we say that the asymptotic gain (AG) property holds.
Definition 5. We say that the system (1) has the limit property (LIM) with respect to $\mathcal{W}$ if there exists $\mu \in \mathcal{K}_{\infty}$ such that for all $x \in M$ and all measurable essentially bounded inputs $d(\cdot)$ the solutions are defined for all $t \geq 0$ and the following holds:

$$
\inf _{t \geq 0}|X(t, x ; d)|_{\mathcal{W}} \leq \mu\left(\|d\|_{\infty}\right) .
$$

Definition 6. We say that the system (1) has the practical global stability (pGS) property with respect to $\mathcal{W}$ if there exist $\beta \in \mathcal{K}_{\infty}$ and $q \geq 0$ such that for all $x \in M$ and all measurable essentially bounded inputs $d(\cdot)$ the following holds for all $t \geq$ 0 :

$$
|X(t, x ; d)|_{\mathcal{W}} \leq q+\beta\left(\max \left\{|x|_{\mathcal{W}},\|d\|_{\infty}\right\}\right) .
$$

It has been shown in [1] that to characterize (3) in terms of Lyapunov functions the following notion is appropriate:

Definition 7. A $\mathcal{C}^{1}$ function $V: M \rightarrow \mathbb{R}$ is a practical ISS-Lyapunov function for (1) if there exists $\mathcal{K}_{\infty}$ functions $\alpha_{1}, \alpha_{2}, \alpha$ and $\gamma$, and scalar $q \geq 0$ and $c \geq 0$ such that

$$
\alpha_{1}\left(|x|_{\mathcal{W}}\right) \leq V(x) \leq \alpha_{2}\left(|x|_{\mathcal{W}}+c\right),
$$

the function $V$ is constant on each $\mathcal{W}_{i}$ and the following dissipative property holds:

$$
D V(x) f(x, d) \leq-\alpha\left(|x|_{\mathcal{W}}\right)+\gamma(|d|)+q .
$$

If the latter inequality holds for $q=0$, then $V$ is said to be an ISS-Lyapunov function.

Notice that the existence of $\alpha_{2}$ and $c$ follows (without any additional assumptions) by standard continuity arguments.

The main result of [1] connecting these robust stability properties is stated below:

Theorem 1. Consider a nonlinear system as in (1) and let a compact invariant set containing all $\alpha$ and $\omega$ limit sets of (2) $\mathcal{W}$ be decomposable (in the sense of Definition 3). Then the following facts are equivalent.

1. The system admits an ISS Lyapunov function;

2. The system enjoys the AG property;

3. The system admits a practical ISS Lyapunov function;

4. The system enjoys the $p A G$ property;

5. The system enjoys the LIM property and the $p G S$.

A system in (1), for which this list of equivalent properties is satisfied, is called ISS with respect to the set $\mathcal{W}$ [1].

\section{Potential Field Method With Static ObStacles}

First, let us consider a simplified model of a mobile agent represented by the integrator dynamics:

$$
\begin{aligned}
\dot{x} & =u_{x}, \\
\dot{y} & =u_{y},
\end{aligned}
$$

where $x \in \mathbb{R}$ and $y \in \mathbb{R}$ are the coordinates of the agent in the plane, $z=\left[\begin{array}{ll}x & y\end{array}\right]^{T}, u_{x} \in \mathbb{R}$ and $u_{y} \in \mathbb{R}$ are the corresponding controls. It is necessary to design the controls $u_{x}, u_{y}$ providing the agent regulation to the origin under avoidance of collisions with isolated point-wise obstacles, which are defined by their coordinates $\zeta_{i}=\left(x_{i}, y_{i}\right)$ and safe distances $d_{i}$ around them for $i=1, \ldots, N$, where $N>0$ is a finite number of obstacles. 
We will assume that $\left|\zeta_{i}-\zeta_{j}\right|>\max \left\{d_{i}, d_{j}\right\}$ and $\left|\zeta_{i}\right|>d_{i}$ for all $1 \leq i \neq j \leq N$, i.e. the obstacles are separated and the origin is not occupied by an obstacle.

The problem will be solved using the potential field method, whose idea consists in defining a repulsion potential $U_{r}$ with respect to the obstacles and attraction potential $U_{a}$ with respect to the origin, next the controls can be designed proportional to the "forces" generated by the total potential $U$, [6]. The main drawback of that approach consists in appearance of local conditional extrema, which theoretically do not allow a global problem solution to be guaranteed by the method. In this work we will use the results presented in the previous section to design the agent dynamics that is $C^{1}$ and ISS with respect to the set $\mathcal{W}$ composed by equilibriums, among them the equilibrium at the origin is attractive, the equilibriums related to the obstacles are repulsing, while ones corresponding to the local extrema are saddle. Next, applying specially designed small perturbations to that ISS system we will avoid the unstable equilibriums.

To design the attraction potential $U_{a}$ we would like to impose the following constraints: it should be twice continuously differentiable with respect to $x$ and $y$, and its gradient should be globally bounded (the velocity of movement out the origin in the collision-free case should be approximately constant in a robotic application). The following potential yields these constraints:

$$
\begin{aligned}
U_{a}(z) & = \begin{cases}|z|^{2} & \text { if }|z| \leq v, \\
|z| & \text { if }|z| \geq \Upsilon, \\
\lambda(|z|)|z|^{2}+[1-\lambda(|z|)]|z| & \text { otherwise, }\end{cases} \\
\lambda(s) & =\left(\frac{2 s^{3}-3(v+\Upsilon) s^{2}+6 \Upsilon v s+\Upsilon^{2}(\Upsilon-3 v)}{\Upsilon^{2}(\Upsilon-3 v)+v^{2}(3 \Upsilon-v)}\right)^{2},
\end{aligned}
$$

where $0<v<\Upsilon<+\infty$ are the design parameters. Thus, the potential $U_{a}$ is quadratic in $z$ close to the origin, it has a linear growth rate far enough and the function $\lambda$ ensures a smooth transition between these zones.

The repulsion potential $U_{r}$ should be also twice continuously differentiable with respect to $x$ and $y$, and it should be active only on a small zone around the obstacle (the agent can detect the obstacle presence only locally in an uncertain environment in a robotic application, for example):

$$
U_{r}(z)=\alpha \sum_{i=1}^{N} \max \left\{0, d_{i}^{2}-\left|z-\zeta_{i}\right|^{2}\right\}^{2},
$$

where $\alpha>0$ is a tuning parameter.

The total potential $U$ has the form:

$$
U(z)=U_{a}(z)+U_{r}(z),
$$

with the gradient

$$
\begin{gathered}
\nabla_{z} U(z)=\nabla_{z} U_{a}(z)+\nabla_{z} U_{r}(z), \\
\nabla_{z} U_{a}(z)= \begin{cases}2 z & \text { if }|z| \leq v, \\
z|z|^{-1} & \text { if }|z| \geq \Upsilon, \\
\varphi(z) & \text { otherwise, }\end{cases} \\
\nabla_{z} U_{r}(z)=-4 \alpha \sum_{i=1}^{N}\left(z-\zeta_{i}\right) \max \left\{0, d_{i}^{2}-\left|z-\zeta_{i}\right|^{2}\right\},
\end{gathered}
$$

where $\varphi(z)=\nabla_{z}\left(\lambda(|z|)|z|^{2}+[1-\lambda(|z|)]|z|\right)$ is the corresponding $C^{1}$ function ensuring a continuous transition between $2 z$ and $z|z|^{-1}$. Note that by construction $\nabla_{z} U(z)$ is a $C^{1}$ function of $z$. As usual in the potential field method we assign:

$$
\left[\begin{array}{l}
u_{x} \\
u_{y}
\end{array}\right]=-\nabla_{z} U(z)+v
$$

where $v \in \mathbb{R}^{2}$ is an auxiliary bounded input to be designed later, then the closed-loop system (4), (5) takes the gradient form:

$$
\dot{z}=-\nabla_{z} U(z)+v .
$$

Next, we are going to show that for $v=0$ this system has an attracting equilibrium at the origin, repulsing equilibriums in a vicinity of $\zeta_{i}$ for each $i=1, \ldots, N$ and a saddle equilibrium in the border of the repulsion zone around $\zeta_{i}$ for each $i=$ $1, \ldots, N$. Therefore, a compact invariant set $\mathcal{W}$ containing all $\alpha$ - and $\omega$-limit sets of (6) for $v=0$ is decomposable in the sense of Definition 3, and that Theorem 1 can be applied to establish ISS with respect to the set $\mathcal{W}$ for the input $v$.

\section{A. Equilibrium at the origin}

Under the restrictions $\left|\zeta_{i}\right|>d_{i}$ for all $1 \leq i \leq N$, the system (6) is reduced to

$$
\dot{z}=-2 z
$$

for $|z| \leq \tilde{v}$ for some $0<\tilde{v} \leq v$, which is obviously locally attractive. For simplicity of presentation below we will assume that the constants $v$ and $\Upsilon$ are selected in a way to provide $\left|\zeta_{i}\right| \geq \Upsilon+d_{i}$ for all $1 \leq i \leq N$.

\section{B. Equilibriums around the obstacles}

Since the obstacles are separated from one another and from the origin, around each obstacle the system (6) takes a reduction $(|z| \geq \Upsilon)$ :

$$
\dot{z}=-z|z|^{-1}+4 \alpha\left(z-\zeta_{i}\right) \max \left\{0, d_{i}^{2}-\left|z-\zeta_{i}\right|^{2}\right\}+v
$$

for some $1 \leq i \leq N$. Clearly, if $d_{i}<\left|z-\zeta_{i}\right|$ then $\dot{z}=-z|z|^{-1}$ and there is no equilibrium, thus we may restrict the attention to the case $\left|z-\zeta_{i}\right| \leq d_{i}$ and

$$
\dot{z}=-z|z|^{-1}+4 \alpha\left(z-\zeta_{i}\right)\left(d_{i}^{2}-\left|z-\zeta_{i}\right|^{2}\right)+v .
$$

On this set the equilibriums of (6) satisfy the vector equation

$$
z=4 \alpha\left(z-\zeta_{i}\right)\left(d_{i}^{2}-\left|z-\zeta_{i}\right|^{2}\right)|z|
$$

or the corresponding scalar equation

$$
\begin{gathered}
|z|^{2}-8 \alpha\left(d_{i}^{2}-\left|z-\zeta_{i}\right|^{2}\right)|z| z^{T}\left(z-\zeta_{i}\right) \\
+16 \alpha^{2}\left|z-\zeta_{i}\right|^{2}\left(d_{i}^{2}-\left|z-\zeta_{i}\right|^{2}\right)^{2}|z|^{2}=0 .
\end{gathered}
$$

Introducing parametrization $z=\kappa \zeta_{i}+\eta$, where $\kappa \in \mathbb{R}$ and $\eta \in \mathbb{R}^{2}$, and substituting it in the last equation it is tedious but straightforward to obtain that for any $|\eta| \neq 0$ the equality is not satisfied. Therefore setup $\eta=0$, then under substitution $z=\kappa \zeta_{i}$ we have

$$
\kappa^{2}\left|\zeta_{i}\right|^{2}\left\{1-4 \alpha\left[d_{i}^{2}-(\kappa-1)^{2}\left|\zeta_{i}\right|^{2}\right]\left|\zeta_{i}\right|(\kappa-1)\right\}^{2}=0,
$$


the equation for equilibriums is reduced to

$$
1-4 \alpha\left[d_{i}^{2}-s^{2}\left|\zeta_{i}\right|^{2}\right]\left|\zeta_{i}\right| s=0
$$

for $s=\kappa-1$, or

$$
s^{3}-\frac{d_{i}^{2}}{\left|\zeta_{i}\right|^{2}} s+\frac{1}{4 \alpha\left|\zeta_{i}\right|^{3}}=0
$$

that is a depressed cubic equation, which by the Cardano's method has only real roots if

$$
\alpha d_{i}^{3}>\frac{3 \sqrt{3}}{8} .
$$

Next, by the Routh-Hurwitz stability criterion the equation has 2 roots with positive real parts. Therefore, for $\left|z-\zeta_{i}\right| \leq$ $d_{i}$ the system (6) has two equilibriums $z_{0}^{i, 1}$ and $z_{0}^{i, 2}$ under the condition (7). The Cardano's method also provides the expressions of exact solutions and, hence, the coordinates of the equilibriums $z_{0}^{i, 1}, z_{0}^{i, 2}$ (not given here for compactness, both equilibriums are located farther from the origin than the obstacle $\zeta_{i}$ on the line connecting the origin and the point $\left.\left(x_{i}, y_{i}\right)\right)$. Finally, the system (6) is continuously differentiable, then the linearization shows that the equilibrium $z_{0}^{i, 1}$ (closer to $\zeta_{i}$ ) is purely repulsing, and another one $z_{0}^{i, 2}$ is saddle (the corresponding local minimum).

To evaluate the zone of repulsion around $\zeta_{i}$ a Lyapunov function for linearization around $z_{0}^{i, 1}$ can be used, or let us consider a Lyapunov function $V(e)=|e|^{2}$ for $e=z-\zeta_{i}$ and $v=0$ :

$$
\begin{aligned}
\dot{V} & =2 e^{T}\left[-z|z|^{-1}+4 \alpha e\left(d_{i}^{2}-|e|^{2}\right)\right] \\
& =-2 e^{T} z|z|^{-1}+8 \alpha V\left(d_{i}^{2}-V\right) \\
& \geq-2|e|+8 \alpha V\left(d_{i}^{2}-V\right) .
\end{aligned}
$$

Note that $|e|=\sqrt{V}$ then

$$
\dot{V} \geq\left[4 \alpha \sqrt{V}\left(d_{i}^{2}-V\right)-1\right] 2 \sqrt{V} .
$$

The Cardano's method can be used to find the solutions of the equation $4 \alpha \sqrt{V}\left(d_{i}^{2}-V\right)=1$, which determines the sign definiteness of $\dot{V}$. The expression in the square brackets $4 \alpha \sqrt{V}\left(d_{i}^{2}-V\right)-1$ reaches its maximum $\frac{2}{\sqrt{3}}\left(\frac{8}{3 \sqrt{3}} \alpha d_{i}^{3}-1\right) d_{i}$ for $V=\frac{1}{3} d_{i}^{2}$, which is positive if the condition (7) is fulfilled (note that since the value of $d_{i}$ is constrained by the physical dimensions of the agent, then (7) is a condition for $\alpha$ to satisfy). Thus the repulsion zone around the obstacle exists and it can be easily estimated.

\section{Robustness with respect to $v$}

The conditions on existence of the equilibriums, established above, are as follows:

Assumption 1. Let the condition (7) be satisfied, $\left|\zeta_{i}-\zeta_{j}\right|>$ $\max \left\{d_{i}, d_{j}\right\}$ and $\left|\zeta_{i}\right| \geq \Upsilon+d_{i}$ for all $1 \leq i \neq j \leq N$.

Now we would like to show that the set $\mathcal{W}=$ $\left\{\{0\}, z_{0}^{1,1}, z_{0}^{1,2}, \ldots, z_{0}^{i, 1}, z_{0}^{i, 2}, \ldots, z_{0}^{N, 1}, z_{0}^{N, 2}\right\}$, which is composed by the equilibrium at the origin and $N$ pairs of equilibriums $z_{0}^{i, 1}, z_{0}^{i, 2}$ associated with each obstacle, contains all $\alpha$ - and $\omega$-limit sets of (6) for $v=0$ and it is decomposable in the sense of Definition 3. The system (6) has a Lyapunov function $U(z)$, by construction $\alpha_{1}(|z|) \leq U(z) \leq \alpha_{2}(|z|)$ for all $z \in \mathbb{R}^{2}$ and some $\alpha_{1}, \alpha_{2} \in \mathcal{K}_{\infty}$, whose derivative has the form:

$$
\begin{aligned}
\dot{U} & =-\left|\nabla_{z} U(z)\right|^{2}+\nabla_{z}^{T} U(z) v \\
& \leq-0.5\left|\nabla_{z} U(z)\right|^{2}+0.5|v|^{2}
\end{aligned}
$$

and the total potential stops to decrease for $v=0$ only on the set where $\nabla_{z} U(z)=0$, but by consideration above it is $\mathcal{W}$ : i.e. there exist $\gamma_{1}, \gamma_{2} \in \mathcal{K}_{\infty}$ such that $\gamma_{1}\left(|z|_{\mathcal{W}}\right) \leq$ $\left|\nabla_{z} U(z)\right| \leq \gamma_{2}\left(|z|_{\mathcal{W}}\right)$ for all $z \in \mathbb{R}^{2}$. There is no cycle in the decomposition of $\mathcal{W}$ due to the same property $\dot{U} \leq 0$ for $v=0$ (indeed the obstacles are separated and to pass from one saddle equilibrium around the obstacle $\zeta_{i}$ to another one around $\zeta_{j}$ it is necessary to cross the zone where $\nabla_{z} U(z)=\nabla_{z} U_{a}(z)$ and $\dot{U}<0$, therefore a trajectory cannot return back). Thus, $\mathcal{W}$ is decomposable and contains all $\alpha$ - and $\omega$-limit sets of (6) for $v=0$. Further,

$$
\dot{U} \leq-0.5 \gamma_{1}^{2}(|z| \mathcal{W})+0.5|v|^{2}
$$

then $U$ is an ISS Lyapunov function and by Theorem 1 the following result has been proven.

Lemma 1. Under Assumption 1 the system (6) is ISS with respect to the set $\mathcal{W}$ for the input $v$.

\section{Design of the input $v$ to escape the local minimums}

The advantage of the ISS property is that appearance of any bounded disturbance $v$ does not lead to the system instability. In our case the total potential function $U$ is also an ISS Lyapunov function for the system (6). If $v=0$ and the agent in (6) is approaching an unstable equilibrium, then according to the expression of $\dot{U}$ the velocity of the agent is decreasing proportionally to $\left|\nabla_{z} U(z)\right|$. Thus, if $\left|\nabla_{z} U(z)\right| \leq \epsilon$ for some predefined $\epsilon>0$ and we are far from the origin, it can be a signal of closeness to a saddle equilibrium, then an input $v \neq 0$ can be generated to shift the movement direction.

The input $v$ can be selected bounded and pushing the system in an arbitrary direction with a uniform distribution, by ISS property the solutions asymptotically will stay close to $\mathcal{W}$. However, using the Lyapunov function $U$ the input $v$ always can be designed in order to additionally guarantee a decreasing of $U$. From (8)

$$
\begin{gathered}
v=\left\{\begin{array}{cc}
\rho\left[\begin{array}{c}
\nabla_{y} U(z) \\
-\nabla_{x} U(z)
\end{array}\right] \quad \text { if }\left|\nabla_{z} U(z)\right| \leq \epsilon \text { and }|z|>v, \\
0 \quad \text { otherwise, }
\end{array}\right. \\
\rho=\operatorname{sgn}\left(y-\frac{y_{i}}{x_{i}} x\right), i=\arg \inf _{1 \leq j \leq N}\left|z-\zeta_{j}\right|, \\
\operatorname{sgn}(s)= \begin{cases}1 & \text { if } s \geq 0, \\
-1 & \text { otherwise }\end{cases}
\end{gathered}
$$

ensures that $\dot{U} \leq 0$ for all $t \geq 0\left(\dot{U}=-\left|\nabla_{z} U(z)\right|^{2}\right.$ while $v \neq 0$ ) and for $U=0$ as well, and for the case of agent velocity dangerous decreasing $\left(\left|\nabla_{z} U(z)\right| \leq \epsilon\right)$ far from the origin $(|z|>v)$ the proposed input $v$ generates an orthogonal disturbance to the current direction of movement. The variable 
$\rho$ defines the orientation of this orthogonal perturbation, in (9) it points out from the line connecting the origin and the point $\left(x_{i}, y_{i}\right)$ (that is the coordinate of the closest obstacle) and where we have the unstable equilibriums.

Assumption 2. Let $z(0) \notin \mathcal{D}=\cup_{i=1}^{N} \mathcal{D}_{i}$ with $\mathcal{D}_{i}=\left\{z \in \mathbb{R}^{2}\right.$ : $\left.\left|z-\zeta_{i}\right| \leq d_{i}\right\}$.

The meaning of Assumption 2 is straightforward: the robot starts in the collision-free condition.

Theorem 2. Under Assumptions 1 and 2 the system (6) with the avoidance control (9) has the origin attractive from all initial conditions $z(0) \notin \mathcal{W} \backslash\{0\}$.

Formally the control (9) does not use ISS property of the set $\mathcal{W}$, it is designed from a pure Lyapunov approach. We may modify (9) as follows in order to make the attractiveness of the origin global:

$$
\begin{gathered}
v=\left\{\begin{array}{cc}
\rho \frac{\varepsilon}{|z|}\left[\begin{array}{c}
y \\
-x
\end{array}\right] \quad \text { if }\left|\nabla_{z} U(z)\right| \leq \epsilon \text { and }|z|>v, \\
0 \quad \text { otherwise, }
\end{array}\right. \\
\rho=\operatorname{sgn}\left(y-\frac{y_{i}}{x_{i}} x\right), i=\arg \inf _{1 \leq j \leq N}\left|z-\zeta_{j}\right|, \\
\operatorname{sgn}(s)= \begin{cases}1 & \text { if } s \geq 0, \\
-1 & \text { otherwise }\end{cases}
\end{gathered}
$$

where $\varepsilon>0$ is a design parameter. It is easy to check that $v^{T} z=0$ for all $z \in \mathbb{R}^{2}$ and $|v|=\varepsilon$ if $\left|\nabla_{z} U(z)\right| \leq \epsilon$ and $|z|>v$ in (10).

Theorem 3. Under Assumption 1 and 2 the system (6) with the avoidance control (10) has the origin globally attractive provided that $\varepsilon>2 \epsilon$ and $\epsilon>0$ is selected sufficiently small.

\section{E. More complex situations}

Of course in reality the assumption about separation between obstacles may be not satisfied, but even for this case the approach can be easily extended. Application of perturbation $v$ with the amplitude $\varepsilon$ does not destroy boundedness of the system trajectories by ISS property. If $\varepsilon$ has been selected sufficiently small, then asymptotically $z(t)$ enters $\mathcal{A}$, as it has been defined above, whose separated subsets contain a single isolated extremum point of $U$. The function $\left|\nabla_{z} U(z)\right|$ is $C^{1}$ by construction, then $\nabla_{z}\left|\nabla_{z} U(z)\right|$ can be calculated and locally $v$ can be selected proportional to $\nabla_{z}\left|\nabla_{z} U(z)\right|$ in order to maximize $\left|\nabla_{z} U(z)\right|$, which is equivalent of the extremum point avoidance. In the simple case presented above the calculation of $\nabla_{z}\left|\nabla_{z} U(z)\right|$ may be avoided.

\section{F. Results of simulation}

For $v=0.1, \Upsilon=0.5, \alpha=2, N=1$ and $\left(x_{1}, y_{1}\right)=$ $(2,2)$ with $d_{1}=1$, the results of the system (6) simulation for different initial conditions with $v=0$ are shown in the left part of Fig. 1. The results of the system (6) simulation with (10) are shown in the right part of Fig 1. As we can conclude, for $v=0$ the potential field method sticks in the local extremum for some initial conditions, while with the
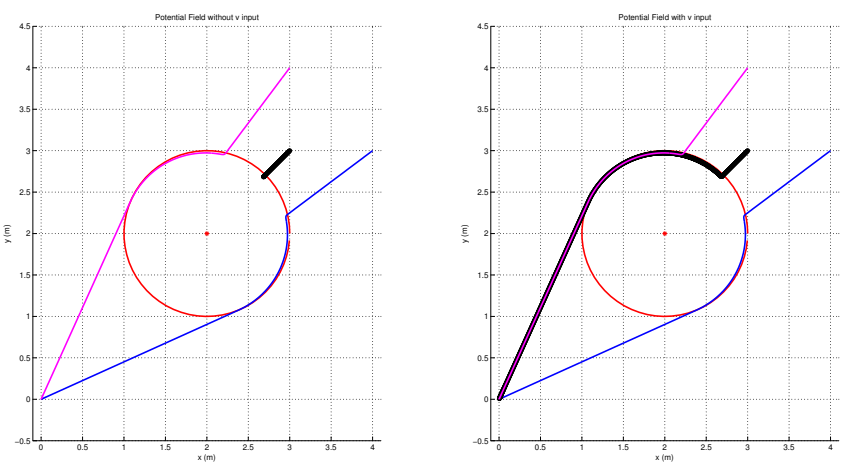

Figure 1: The results of the system (6) simulation

proposed modifications (9) or (10) the origin is attractive under provided restrictions.

\section{WHEELED MOBILE ROBOT REGULATION WITH COLLISION AVOIDANCE}

Consider a wheeled mobile robot, whose model is given by a unicycle:

$$
\begin{aligned}
& \dot{q}_{x}=\cos (\theta) u\left(1+\delta_{1}\right), \\
& \dot{q}_{y}=\sin (\theta) u\left(1+\delta_{1}\right), \\
& \dot{q}_{\theta}=\omega\left(1+\delta_{2}\right),
\end{aligned}
$$

where $\left(q_{x}, q_{y}\right) \in \mathbb{R}^{2}$ is the robot position, $q_{\theta} \in(-\pi, \pi]$ is the robot orientation, $|u| \leq u_{\max }$ and $|\omega| \leq \omega_{\max }$ are linear and angular velocities of the robot respectively $\left(u_{\max }\right.$ and $\omega_{\max }$ are given bounds), $\delta_{k} \in\left[\delta_{\min }, \delta_{\max }\right], k=1,2$ are exogenous bounded disturbances [3], which are introduced in order to represent the dynamical model uncertainties/dynamics (they are not taken into account in the classical unicycle model), $-1<\delta_{\min }<\delta_{\max }<+\infty$.

In order to apply the approach developed for the system (6) with the control (9) or (10), as usual in the potential field method, the trajectory generated by (6), (10) (or with (9)) can be used as a reference for (11), defining $\theta_{d}=$ $\operatorname{atan} 2\left(\nabla_{y} U(z), \nabla_{x} U(z)\right)-q_{\theta}$ :

$$
\begin{aligned}
u & =\frac{u_{\max }}{1+\varepsilon} \sqrt{u_{x}^{2}+u_{y}^{2}}, \\
\omega & =\omega_{\max } \sqrt{\left|\theta_{d}\right|} \operatorname{sign}\left(\theta_{d}\right) .
\end{aligned}
$$

The results of simulation of the system (11), (12) are shown in Fig. 2 for $u_{\max }=1$ and $\omega_{\max }=3$ in the case of single obstacle (Fig.2a) and multiple obstacles (Fig. 2b). In both figures of Fig. 2 the obstacle is the zone filled in violet while the safe distance is the black circle around the obstacle itself. The method is called NON-Apf to emphasize the non asymptotic (finite time) behavior of the controller acting on the orientation of the WMR. Moreover the proposed modification has been compared with the standard APF [13] to show how the improvement of this work allows the WMR also to keep a bigger distance from the obstacle(s).

\section{A. More complex scenarios}

Several simulations were run also for more complex scenarios, in which the features of the equipped sensor for an 


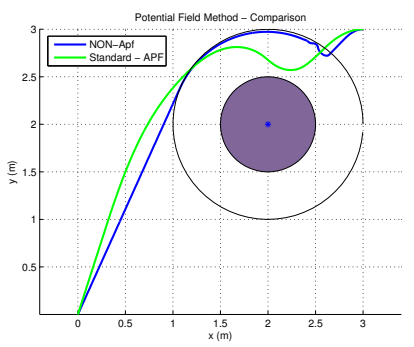

(a) Single obstacle

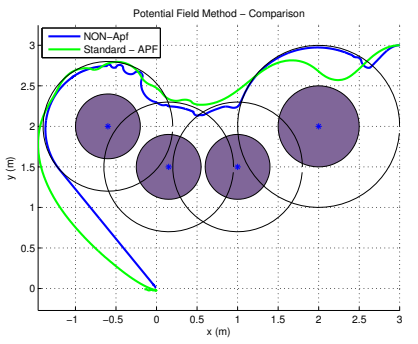

(b) Several obstacles
Figure 2: The results of simulation of (11), (12)

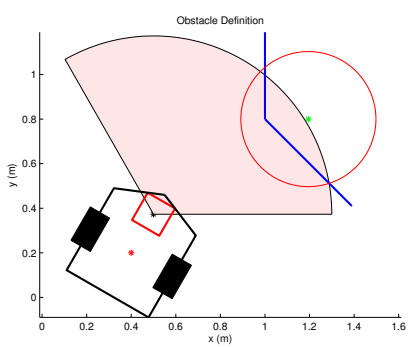

(a) WMR equipped with a LIDAR laser range finder: obstacle definition

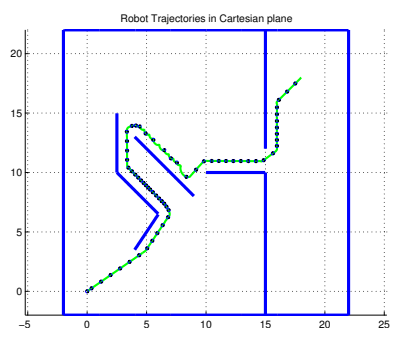

(b) The path followed by the WMR in a complex scenario
Figure 3: Results on complex environment

eventual implementation are taken into account (i.e. a LIDAR laser ranger finder). In Fig. $3 \mathrm{~b}$ is shown the path followed by the WMR using the proposed modification of the potential field method, while in Fig. 3a the strategy to decide which is the "point" to use as reference for the obstacle. Basically, the chosen point $\zeta$, green star in Fig. 3a, is the averaging on the $x$ and $y$ coordinates of the LIDAR sensed points in a predefined range, in the test $1 \mathrm{~m}$; the radius is the distance among $\zeta$ itself and the farthest sensed point of the scan, which leads to the definition of the influence distance $d$ that is the radius augmented of the radius of the robot.

\section{COnClusions}

The modification of the PF method based on the ISS property for decomposable invariant sets was shown to be able to avoid local minima, main issue of the standard solution. Furthermore, it has been formally shown how the introduction of the $v$ input does not introduce new equilibriums for the system making the origin (target) the only attractive point. When coupled with the finite time control for the orientation for a non-holonomic WMR it showed a nice behavior avoiding single obstacles, multiple obstacles and in complex environments.

The authors intend to improve the method to cancel any oscillations in future formulations and to extend it to be used in the case of multi-agent systems.

\section{ACKNOWLEGMENT}

This work was supported in part by Région Nord-Pas de Calais, by the Government of Russian Federation (Grant 074-
U01) and the Ministry of Education and Science of Russian Federation (Project 14.Z50.31.0031).

\section{REFERENCES}

[1] D. Angeli and D. Efimov. On input-to-state stability with respect to decomposable invariant sets. In Proc. 52nd IEEE Conference on Decision and Control, Florence, 2013.

[2] C.I Connolly, J. B. Burns, and R. Weiss. Path planning using laplace's equation. In Robotics and Automation, 1990. Proceedings., 1990 IEEE International Conference on, pages 2102-2106 vol.3, May 1990.

[3] Guerra M. Efimov D. Zheng G. and Perruquetti W. Finite-time supervisory stabilization for a class of nonholonomic mobile robots under input disturbances. In 19th IFAC World Congress, Cape Town, South Africa, pages 4867-4872, Aug 2014.

[4] S.S. Ge and Y.J. Cui. New potential functions for mobile robot path planning. Robotics and Automation, IEEE Transactions on, 16(5):615620 , Oct 2000.

[5] J. Guckenheimer and P. Holmes. Structurally stable heteroclinic cycles. Math. Proc. Camb. Phil. Soc., 103:189-192, 1988.

[6] O. Khatib. Real-time obstacle avoidance for manipulators and mobile robots. In IEEE International Conference on Robotics and Automation, pages 500-505, St. Louis, Missouri, 1985.

[7] D.H. Kim, H.O. Wang, Guohua Ye, and Seiichi Shin. Decentralized control of autonomous swarm systems using artificial potential functions: analytical design guidelines. In Decision and Control, 2004. CDC. 43rd IEEE Conference on, volume 1, pages 159-164, Dec 2004.

[8] Y. Koren and J. Borenstein. Potential field methods and their inherent limitations for mobile robot navigation. In Proc. IEEE Conf. Robotics and Automation, pages 1398-1404, Sacramento, CA, 1991.

[9] AA Masoud. A harmonic potential field approach for navigating a rigid, nonholonomic robot in a cluttered environment. In Robotics and Automation, 2009. ICRA '09. IEEE International Conference on, pages 3993-3999, May 2009.

[10] Z. Nitecki and M. Shub. Filtrations, decompositions, and explosions. American Journal of Mathematics, 97(4):1029-1047, 1975.

[11] Makiko Okamoto and Maruthi R. Akella. Novel potential-functionbased control scheme for non-holonomic multi-agent systems to prevent the local minimum problem. International Journal of Systems Science, 0(0):1-15, 2013.

[12] Min Gyu Park and Min Cheol Lee. Real-time path planning in unknown environment and a virtual hill concept to escape local minima. In Industrial Electronics Society, 2004. IECON 2004. 30th Annual Conference of IEEE, volume 3, pages 2223-2228 Vol. 3, Nov 2004.

[13] E. Rimon and D.E. Koditschek. Exact robot navigation using artificial potential functions. Robotics and Automation, IEEE Transactions on, 8(5):501-518, Oct 1992.

[14] Keisuke Sato. Deadlock-free motion planning using the laplace potential field. Advanced Robotics, 7(5):449-461, 1992.

[15] Yingchong M. Zheng G. Perruquetti W. and Z. Qiu. Motion planning for non-holonomic mobile robots using the i-pid controller and potential field. In Intelligent Robots and Systems (IROS), 2014 IEEE/RSJ International Conference on, Sep 2014. 